\title{
Direct comparison of in-vivo and post-mortem spin-echo based diffusion tensor imaging in the porcine heart
}

\author{
Christian T Stoeck ${ }^{1,2^{*}}$, Constantin von Deuster ${ }^{1,2}$, Nikola Cesarovic ${ }^{3}$, Martin Genet', Maximilian Y Emmert ${ }^{3,4}$, \\ Sebastian Kozerke ${ }^{1,2}$
}

From 18th Annual SCMR Scientific Sessions

Nice, France. 4-7 February 2015

\section{Background}

Spin-echo based cardiac diffusion tensor imaging (DTI) is highly sensitive to myocardial strain [1]. Imaging during systolic contraction requires precise planning of the sequence timing [2]. Second order motion compensated diffusion encoding has recently been proposed for small animal imaging [3] to reduce the impact of myocardial strain on the diffusion tensor.

It is the objective of the present work to compare second order motion compensated spin-echo DTI of the in-vivo and post-mortem porcine heart on a clinical MR system.

\section{Methods}

Second order motion compensated diffusion encoding gradients were incorporated into a cardiac triggered single-shot spin-echo sequence (Figure 1). A pig (55kg) was imaged on a $1.5 \mathrm{~T}$ clinical system (Philips Healthcare, Best, The Netherlands) equipped with a gradient system delivering $80 \mathrm{mT} / \mathrm{m} @ 100 \mathrm{mT} / \mathrm{m} / \mathrm{ms}$. Eight slices (Figure 2a) were acquired during free breathing with the following parameters: resolution: $2.2 \times 2.2 \mathrm{~mm}^{2}$, slice thickness: $6 \mathrm{~mm}$, reduced FOV [4]: $230 \times 98 \mathrm{~mm}^{2}$, TR/TE: 2R-R/83ms, 10 averages. Fat suppression was established by spectral-spatial excitation. Ten diffusion-encoding directions [5] with a b-value of $450 \mathrm{~s} / \mathrm{mm}^{2}$ were applied during early systole. The pig was euthanized by a potassium injection inside the MR scanner and the imaging protocol was repeated. Helix angle maps were calculated upon tensor reconstruction [6]. The myocardium was

${ }^{1}$ Institute for Biomedical Engineering, University and ETH Zurich, Zurich, Switzerland

Full list of author information is available at the end of the article segmented in 4 angular and 4 radial segments per slice similar to the procedure proposed in [7] (Figure 2b).

\section{Results}

Example helix angle maps are shown in Figure 2a). Sectors were spatially matched and the corresponding correlation analysis for in-vivo vs. post-mortem data are presented in Figure 2c). Root mean squared differences between sectors in-vivo and post-mortem were $10.0^{\circ}, 8.9^{\circ}$ and $11.7^{\circ}$ for basal, mid and apical levels, respectively. Despite significant deformation of the post-mortem heart due to the loss in blood pressure, good agreement between in-vivo and post-mortem data is revealed.

\section{Conclusions}

Good correlation between in-vivo and post-mortem imaging was found proving that bulk motion and strain effects are well suppressed if second order motion compensated diffusion gradients are employed.

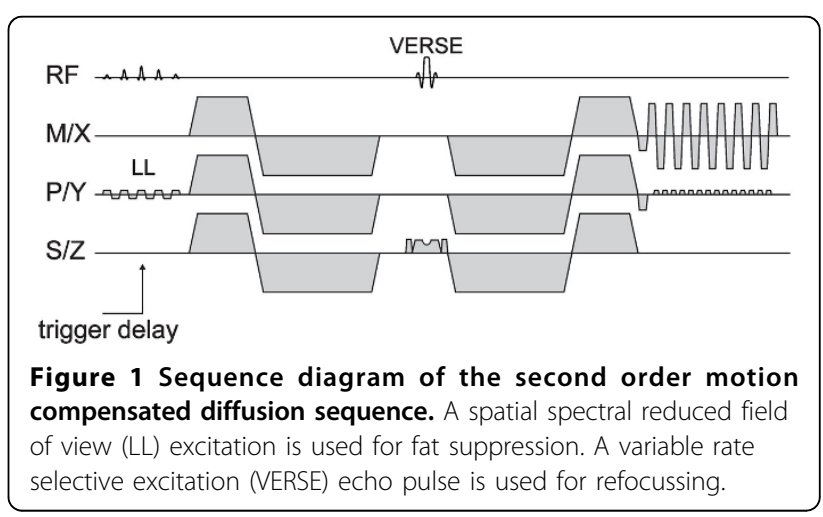




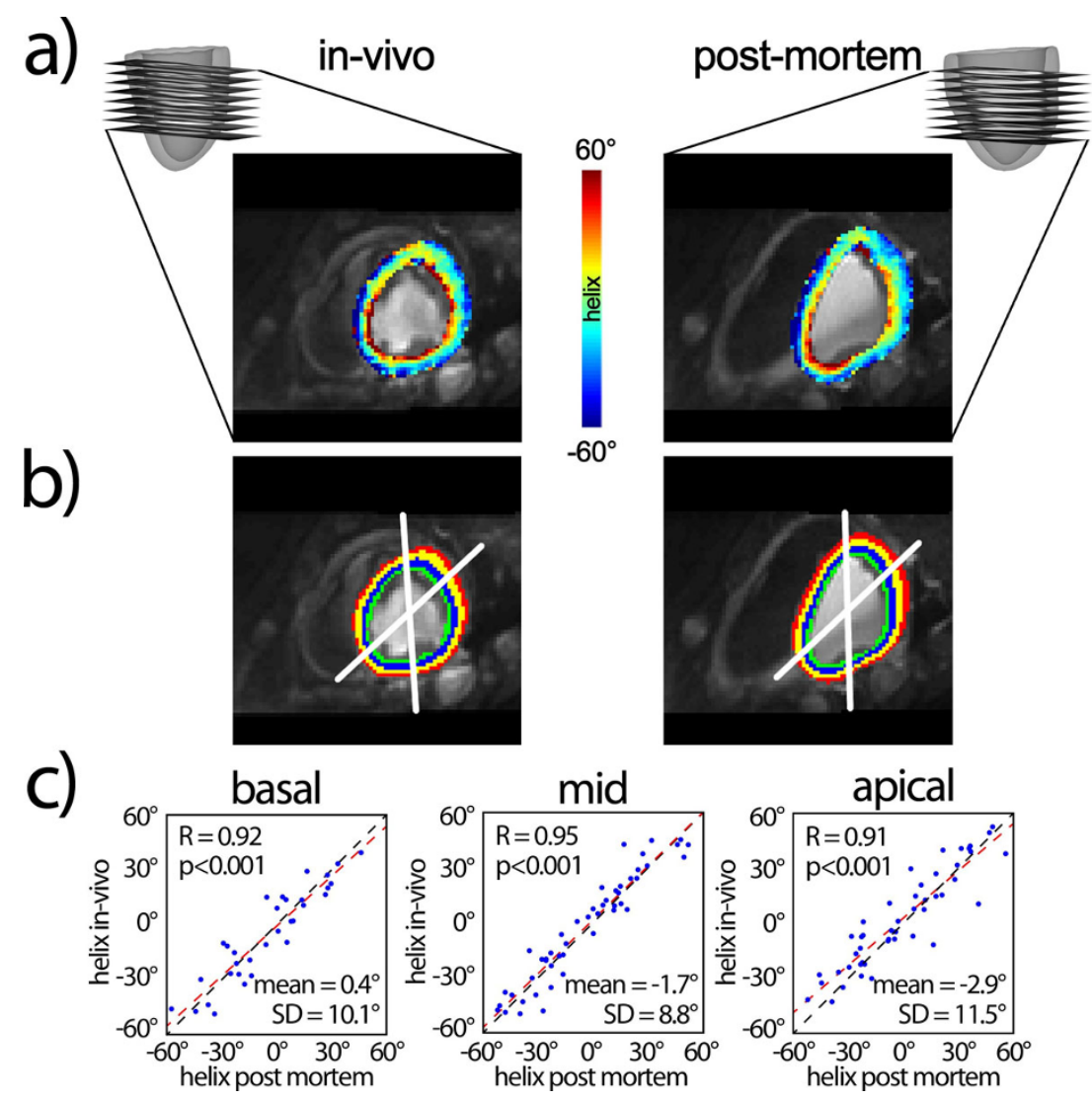

Figure 2 Slice distribution and example helix angle maps for in-vivo and post-mortem imaging are shown in a). Four angular and four radial sectors b) were identified. Mean helix angles per sector were spatially matched and correlation analysis is shown in c). The mean and one standard deviation (SD) of the differences between in-vivo and post mortem are reported.

\section{Funding}

EU FP7 Marie-Curie fellowship to MG.

\section{Authors' details}

'Institute for Biomedical Engineering, University and ETH Zurich, Zurich, Switzerland. ${ }^{2}$ Imaging Sciences and Biomedical Engineering, King's College London, London, UK. ${ }^{3}$ Department of Surgical Research, University Hospital Zurich, Zurich, Switzerland. ${ }^{4}$ Clinic for Cardiovascular Surgery, University Hospital Zurich, Zurich, Switzerland.

Published: 3 February 2015

\section{References}

1. Gamper, et al: MRM 2007.

2. Stoeck, et al: ISMRM 2011.

3. Welsh, et al: ISMRM 2014.

4. Feinberg, et al: Radiology 1985.

5. Jones, et al: MRM 1999.

6. Stoeck, et al: PLOS ONE 2014

7. Nielles-Vallespin, et al: MRM 2013.

doi:10.1186/1532-429X-17-S1-P76

Cite this article as: Stoeck et al:: Direct comparison of in-vivo and postmortem spin-echo based diffusion tensor imaging in the porcine heart. Journal of Cardiovascular Magnetic Resonance 2015 17(Suppl 1):P76.

Submit your next manuscript to BioMed Central and take full advantage of:

- Convenient online submission

- Thorough peer review

- No space constraints or color figure charges

- Immediate publication on acceptance

- Inclusion in PubMed, CAS, Scopus and Google Scholar

- Research which is freely available for redistribution 